\title{
Transcriptional Signatures, Imaging, and Coronary Artery Disease Diagnosis
}

\author{
Steven Rosenberg ${ }^{1}$
}

Received: 29 April 2015 / Accepted: 4 May 2015 / Published online: 13 May 2015

(C) Springer Science+Business Media New York 2015

Coronary artery disease and its sequelae including myocardial infarction are a major source of morbidity and mortality. The likelihood of obstructive coronary artery disease (CAD) development in individuals is substantially heritable (30-60\% or more) [1], although very large genome-wide association studies examining common single nucleotide polymorphisms (SNPs) such as the CardioGRAM studies have only accounted for $10 \%$ even with sophisticated statistical approaches [2]. Thus, despite a very large scientific focus on the genetics of CAD, much is still unknown. Coronary atherosclerosis is characterized by a long-term maladaptive inflammatory immune response [3, 4] and complex interactions with multiple organs and tissues. Thus, it might be expected that biological variables which integrate both genetics and environment, such as examination of RNA and protein expression in circulating blood cells or plasma, might provide a fruitful avenue for discovery and development of signatures for the diagnosis of the presence or absence of CAD. Since circulating cell-based RNA signatures may reflect both the development of, and response to, atherosclerotic plaque, their utility is particularly likely given the systemic nature of atherosclerosis. An enhanced role for atherosclerotic disease burden rather than socalled vulnerable plaque in cardiovascular event prognosis, even in the case of non-obstructive CAD, also supports this hypothesis [5, 6], and the disregulation of RNA expression in circulating cells appears to correlate with the burden of atherosclerosis [7, 8]. In addition, extracellular RNAs, especially microRNAs, as well as long non-coding RNAs may also be of diagnostic utility $[9,10]$. Finally, recent analysis of the relative global importance of transcriptional and translational

Editor-in-Chief Jennifer L. Hall oversaw the review of this article

Steven Rosenberg

srosenberg@cardiodx.com

1 CardioDx, Inc., Redwood City, CA, USA regulation of protein expression has suggested that the former has been underestimated in prior work and that transcriptional regulation accounts for a large majority of variation in the expressed genome, reinforcing the importance of examining RNA levels [11-13].

In this issue of JCTR a series of papers on the state of the art in whole blood transcriptional signatures and imaging for CAD and CAD risk factors are presented. Kraus and coworkers describe the establishment, characteristics, and track record of CATHGEN, one of the first and largest biorepositories for CAD that collected suitable samples for DNA, cellular and extracellular RNA, proteomic, and metabolomics analyses in a population referred for cardiac catheterization. Wingrove and Rhees take us through examples of methods required to discover, develop, and validate whole blood RNA signatures, utilizing in part, samples from the CATHGEN biorepository described by Kraus et al., and leading to the commercialization of a CAD diagnostic classifier comprised of sex-specific age-dependent risk functions and expression levels of 23 genes [14, 15]. Friede and colleagues describe how genomic-based RNA classifiers could be used in clinical practice, including those for diagnosis and prognosis of CAD, as well as novel whole blood gene expression classifiers for aspirin responsiveness and cardiovascular risk factors such as smoking. Finally, Li and colleagues discuss how CT-angiography coupled with genetics could enhance CAD prognosis in a synergistic manner.

\section{References}

1. Deloukas, P., Kanoni, S., Willenborg, C., Farrall, M., Assimes, T. L., Thompson, J. R., Ingelsson, E., Saleheen, D., Erdmann, J., Goldstein, B. A., Stirrups, K., Konig, I. R., Cazier, J. B., Johansson, A., Hall, A. S., Lee, J. Y., Willer, C. J., Chambers, J. C., Esko, T., Folkersen, L., Goel, A., Grundberg, E., Havulinna, A. S., Ho, W. K., Hopewell, J. C., Eriksson, N., Kleber, M. E., Kristiansson, K., Lundmark, P., Lyytikainen, L. P., Rafelt, S., 
Shungin, D., Strawbridge, R. J., Thorleifsson, G., Tikkanen, E., Van Zuydam, N., Voight, B. F., Waite, L. L., Zhang, W., Ziegler, A., Absher, D., Altshuler, D., Balmforth, A. J., Barroso, I., Braund, P. S., Burgdorf, C., Claudi-Boehm, S., Cox, D., Dimitriou, M., Do, R., Doney, A. S., El Mokhtari, N., Eriksson, P., Fischer, K., Fontanillas, P., Franco-Cereceda, A., Gigante, B., Groop, L., Gustafsson, S., Hager, J., Hallmans, G., Han, B. G., Hunt, S. E., Kang, H. M., Illig, T., Kessler, T., Knowles, J. W., Kolovou, G., Kuusisto, J., Langenberg, C., Langford, C., Leander, K., Lokki, M. L., Lundmark, A., McCarthy, M. I., Meisinger, C., Melander, O., Mihailov, E., Maouche, S., Morris, A. D., Muller-Nurasyid, M., Nikus, K., Peden, J. F., Rayner, N. W., Rasheed, A., Rosinger, S., Rubin, D., Rumpf, M. P., Schafer, A., Sivananthan, M., Song, C., Stewart, A. F., Tan, S. T., Thorgeirsson, G., van der Schoot, C. E., Wagner, P. J., Wells, G. A., Wild, P. S., Yang, T. P., Amouyel, P., Arveiler, D., Basart, H., Boehnke, M., Boerwinkle, E., Brambilla, P., Cambien, F., Cupples, A. L., de Faire, U., Dehghan, A., Diemert, P., Epstein, S. E., Evans, A., Ferrario, M. M., Ferrieres, J., Gauguier, D., Go, A. S., Goodall, A. H., Gudnason, V., Hazen, S. L., Holm, H., Iribarren, C., Jang, Y., Kahonen, M., Kee, F., Kim, H. S., Klopp, N., Koenig, W., Kratzer, W., Kuulasmaa, K., Laakso, M., Laaksonen, R., Lee, J. Y., Lind, L., Ouwehand, W. H., Parish, S., Park, J. E., Pedersen, N. L., Peters, A., Quertermous, T., Rader, D. J., Salomaa, V., Schadt, E., Shah, S. H., Sinisalo, J., Stark, K., Stefansson, K., Tregouet, D. A., Virtamo, J., Wallentin, L., Wareham, N., Zimmermann, M. E., Nieminen, M. S., Hengstenberg, C., Sandhu, M. S., Pastinen, T., Syvanen, A. C., Hovingh, G. K., Dedoussis, G., Franks, P. W., Lehtimaki, T., Metspalu, A., Zalloua, P. A., Siegbahn, A., Schreiber, S., Ripatti, S., Blankenberg, S. S., Perola, M., Clarke, R., Boehm, B. O., O'Donnell, C., Reilly, M. P., Marz, W., Collins, R., Kathiresan, S., Hamsten, A., Kooner, J. S., Thorsteinsdottir, U., Danesh, J., Palmer, C. N., Roberts, R., Watkins, H., Schunkert, H., \& Samani, N. J. (2013). Large-scale association analysis identifies new risk loci for coronary artery disease. Nature Genetics, 45, 25-33.

2. Tada, H., Won, H. H., Melander, O., Yang, J., Peloso, G. M., \& Kathiresan, S. (2014). Multiple associated variants increase the heritability explained for plasma lipids and coronary artery disease. Circulation Cardiovascular Genetics, 7, 583-587.

3. Hansson, G. K., \& Libby, P. (2006). The immune response in atherosclerosis: a double-edged sword. Nature Reviews Immunology, 6, 508-519.

4. Hansson, G. K. (2005). Inflammation, atherosclerosis, and coronary artery disease. The New England Journal of Medicine, 352, $1685-1695$.

5. Arbab-Zadeh, A., \& Fuster, V. (2015). The myth of the "vulnerable plaque": transitioning from a focus on individual lesions to atherosclerotic disease burden for coronary artery disease risk assessment. Journal of the American College of Cardiology, 65, 846-855.

6. Chow, B. J., Small, G., Yam, Y., Chen, L., McPherson, R., Achenbach, S., Al-Mallah, M., Berman, D. S., Budoff, M. J., Cademartiri, F., Callister, T. Q., Chang, H. J., Cheng, V. Y., Chinnaiyan, K., Cury, R., Delago, A., Dunning, A., Feuchtner,
G., Hadamitzky, M., Hausleiter, J., Karlsberg, R. P., Kaufmann, P. A., Kim, Y. J., Leipsic, J., LaBounty, T., Lin, F., Maffei, E., Raff, G. L., Shaw, L. J., Villines, T. C., \& Min, J. K. (2015). Prognostic and therapeutic implications of statin and aspirin therapy in individuals with nonobstructive coronary artery disease: results from the confirm (coronary ct angiography evaluation for clinical outcomes: an international multicenter registry) registry. Arteriosclerosis, Thrombosis, and Vascular Biology, 35, 981-989.

7. Voros, S., Elashoff, M. R., Wingrove, J. A., Budoff, M. J., Thomas, G. S., \& Rosenberg, S. (2014). A peripheral blood gene expression score is associated with atherosclerotic plaque burden and stenosis by cardiovascular CT-angiography: results from the predict and compass studies. Atherosclerosis, 233, 284-290.

8. Wingrove, J. A., Daniels, S. E., Sehnert, A. J., Tingley, W., Elashoff, M. R., Rosenberg, S., Buellesfeld, L., Grube, E., Newby, L. K., Ginsburg, G. S., \& Kraus, W. E. (2008). Correlation of peripheral-blood gene expression with the extent of coronary artery stenosis. Circulation Cardiovascular Genetics, 1, 31-38.

9. Stellos, K., \& Dimmeler, S. (2014). Vascular microRNAs: from disease mechanisms to therapeutic targets. Circulation Research, 114, 3-4.

10. Uchida, S., \& Dimmeler, S. (2015). Long noncoding RNAs in cardiovascular diseases. Circulation Research, 116, 737-750.

11. Battle, A., Khan, Z., Wang, S. H., Mitrano, A., Ford, M. J., Pritchard, J. K., \& Gilad, Y. (2015). Genomic variation. Impact of regulatory variation from RNA to protein. Science (New York, N.Y.), 347, 664-667.

12. Jovanovic, M., Rooney, M. S., Mertins, P., Przybylski, D., Chevrier, N., Satija, R., Rodriguez, E. H., Fields, A. P., Schwartz, S., Raychowdhury, R., Mumbach, M. R., Eisenhaure, T., Rabani, M., Gennert, D., Lu, D., Delorey, T., Weissman, J. S., Carr, S. A., Hacohen, N., \& Regev, A. (2015). Immunogenetics. Dynamic profiling of the protein life cycle in response to pathogens. Science (New York, N.Y.), 347, 1259038.

13. Li, J. J., \& Biggin, M. D. (2015). Gene expression. Statistics requantitates the central dogma. Science (New York, N.Y.), 347, 1066-1067.

14. Rosenberg, S., Elashoff, M. R., Beineke, P., Daniels, S. E., Wingrove, J. A., Tingley, W. G., Sager, P. T., Sehnert, A. J., Yau, M., Kraus, W. E., Newby, L. K., Schwartz, R. S., Voros, S., Ellis, S. G., Tahirkheli, N., Waksman, R., McPherson, J., Lansky, A., Winn, M. E., Schork, N. J., \& Topol, E. J. (2010). Multicenter validation of the diagnostic accuracy of a blood-based gene expression test for assessing obstructive coronary artery disease in nondiabetic patients. Annals of Internal Medicine, 153, 425-434.

15. Thomas, G. S., Voros, S., McPherson, J. A., Lansky, A. J., Winn, M. E., Bateman, T. M., Elashoff, M. R., Lieu, H. D., Johnson, A. M., Daniels, S. E., Ladapo, J. A., Phelps, C. E., Douglas, P. S., \& Rosenberg, S. (2013). A blood-based gene expression test for obstructive coronary artery disease tested in symptomatic nondiabetic patients referred for myocardial perfusion imaging the compass study. Circulation Cardiovascular Genetics, 6, 154-162. 